\title{
miR-204-5p inhibits the occurrence and development of osteoarthritis by targeting Runx2
}

\author{
JIAQING CAO, XINYOU HAN, XIN QI, XIANGYUN JIN and XIAOLIN LI \\ Department of Orthopedic Surgery, The Sixth People's Hospital Affiliated to \\ Shanghai Jiao Tong University, Shanghai 200233, P.R. China
}

Received September 26, 2017; Accepted July 23, 2018

DOI: $10.3892 / \mathrm{ijmm} .2018 .3811$

\begin{abstract}
One of the hallmarks of osteoarthritis (OA) development is endochondral ossification, in which Runt-related transcription factor-2 (Runx2) is aberrantly expressed. Runx2 was previously identified to be regulated by microRNA-204-5p (miR-204-5p). The aim of the present study was to investigate the potential function of miR-204-5p regulating Runx2 during the development of OA and the underlying molecular mechanism. The expression levels of miR-204-5p and Runx2 were determined in tissue specimens. Rat OA models were established by transecting the anterior and posterior cruciate ligaments and removing the meniscus. Rats were treated with miR-204-5p agomir and miR-204-5p negative control (NC). All in vitro experiments were performed using rat primary chondrocytes and the SW-1353 human bone chondrosarcoma cell line. It was identified that the expression of miR-204-5p was significantly decreased, whereas Runx 2 was significantly increased, in human OA tissues compared with in non-OA tissues, and levels were inversely associated with each other in primary chondrocytes and chondrosarcoma cells. Overexpression of miR-204-5p decreased the proliferation of chondrocytes and SW-1353 cells. Using a luciferase reporter assay, Runx 2 was identified to be a direct target of miR-204-5p in chondrocytes and overexpressed miR-204-5p altered the expression of collagens II, X and matrix metalloproteinase (MMP)-1 and MMP-13 in primary chondrocytes and SW-1353 cells. Histological analysis revealed that miR-204-5p treatment ameliorated the OA-like phenotype that is reflected by assessment of cartilage thickness and Mankin's score. Runx 2 expression was gradually increased as the rats increased in age. At 10 weeks of miR-204-5p agomir treatment, altered expression levels of collagens II and X, cartilage oligomeric
\end{abstract}

Correspondence to: Dr Xiaolin Li, Department of Orthopedic Surgery, The Sixth People's Hospital Affiliated to Shanghai Jiao Tong University, 600 Yishan Road, Xuhui, Shanghai 200233, P.R. China

E-mail: lixiaolin@sjtu.edu.cn

Key words: microRNA-204-5p, Runt-related transcription factor-2, osteoarthritis, chondrocyte proliferation matrix protein fragment, aggrecan, MMP-1 and MMP-13 were observed in the treatment group compared with the NC group. In conclusion, the results of the present study indicated that miR-204-5p decreases chondrocyte proliferation and ameliorates the OA-like phenotype in rats with surgically induced OA by targeting Runx 2 .

\section{Introduction}

Chondrocytes, the cellular component of human articular cartilage, serve an important function in maintaining the matrix components under normal conditions (1). In healthy articular cartilage, chondrocytes resist proliferation and terminal differentiation. By contrast, chondrocytes in diseased cartilage progressively proliferate and develop hypertrophy (2). Differentiation of chondrocytes triggers a disturbed cartilage homeostasis favoring articular cartilage degradation and formation of osteophytes.

Gene expression during chondrocyte maturation is controlled by transcription factors. Runt-related transcription factor 2 (Runx2) is one of the central positive regulators within the transition phase from proliferating to hypertrophic chondrocytes (2). It is responsible for proliferation and differentiation of chondrocytes, and its loss contributes to chondroclast development by regulating the nuclear factor $\kappa \mathrm{B}$ signaling pathways (3). Runx $2 / 3$ double-deficient mice lack hypertrophic chondrocytes in all parts of the skeleton (4). Runx 2 is overexpressed in OA cartilages and implicated in DNA methylation $(5,6)$, and enhancement of Runx 2 stimulates hypertrophic chondrocyte differentiation (7).

MicroRNAs (miRNAs/miRs) are small non-coding RNAs that regulate gene expression by binding to 3'-untranslated regions (3'-UTRs) of specific mRNA of target genes (8). The function of miRNAs has been identified to be associated with a variety of inflammatory pathogenesis, including cartilage disease and chondrogenesis (9). It was demonstrated that miR-204-5p is sponged by the long non-coding RNA (lncRNA) taurine upregulated 1 to promote osteoblast differentiation by upregulating Runx2 (10). In osteogenesis of rat bone marrow mesenchymal stem cells, the IncRNA TCONS_00041960 was identified to interact with miR-204-5p to regulate Runx2 (11). In mesenchymal progenitor cells, chondrogenic and osteogenic cell lines, it has also been identified that miR-204 is able to bind to the 3 '-UTR of the Runx2 gene and regulate its 
expression $(12,13)$. Another study identified that, among the genes involved in osteoblast and osteoclast activity, changes in miRNAs are involved in osteoblastogenesis, including miR-204-5p, but Runx 2 was unchanged in patients with clinically and biochemically confirmed acromegaly (14). However, to the best of our knowledge, no previous study has investigated the potential function of miR-204-5p and its association with Runx 2 in OA. Therefore, we hypothesized that there may be a regulation between miR-204-5p and Runx2 in OA development, and, if so, investigate how the axis of miR-204-5p/Runx2 may function. The aim of the present study was to investigate the effect of miR-204-5p in OA cells and rats with OA, as well as the underlying molecular mechanism.

\section{Materials and methods}

Human tissue specimens and ethical statements. Normal adult articular cartilage were obtained from patients with idiopathic osteonecrosis of femoral condyle ( $n=7 ; 3$ males and 4 females; age range, 63-75 years) who did not receive radiographic or any intra-articular treatment. Cartilages of the femoral head and knee joint were obtained from patients with OA who underwent total knee arthroplasty $(n=10 ; 4$ males and 6 females; age range, 59-77 years). Since cartilage degradation and/or osteophyte formation are the characteristics of OA development, necrotic bone tissues were selected as negative control (NC), in which cartilage degradation or osteophyte formation cannot be observed. The samples were then stored at $-80^{\circ} \mathrm{C}$ until analysis. All human studies were approved by the institutional ethics review board of The Sixth People's Hospital Affiliated to Shanghai Jiao Tong University (Shanghai, China). Written informed consent was obtained from each patient.

Animal models. All animal procedures were approved by the Animal Care and Use Committee of the Sixth People's Hospital Affiliated to Shanghai Jiao Tong University. In total, 45 12-week-old male Sprague-Dawley (SD) rats (weighing 200-300 g) (Shanghai Laboratory Animal Center, Shanghai, China) were housed in groups of two under a 12-h light/12-h dark cycle at room temperature with food and water ad libitum. All animals were randomly divided into three groups: OA miR-204-5p agomir treatment group $(n=15)$, OA $\mathrm{NC}$ group $(n=15)$ and sham surgery group $(n=15)$. The rats in the miR-204-5p agomir treatment or NC group were subjected to one-time injection of miR-204-5p agomir ( $800 \mathrm{pmol})$ or NC $(800 \mathrm{pmol})$ into the joint $6 \mathrm{~h}$ before surgery, and 5 weeks later the same volume of the compounds was injected again. Rats were sacrificed at 5, 10 and 15 weeks after surgery. The OA model was established by transecting the anterior and posterior cruciate ligaments and removing the meniscus as described previously (15). Sham surgery consisted of all aforementioned steps except for ligament transection.

Isolation and culture of cells. Primary chondrocytes were isolated from newborn SD rats as described previously (16). Briefly, following sacrifice of the rats, the articular cartilage of the knee joint was removed, and attached muscle, perichondrium and connective tissue were removed. The cartilages were shredded, washed in PBS and digested with trypsin for at $37^{\circ} \mathrm{C}$. Subsequently, they were washed with PBS and digested with type II collagenase solution. Dissociated cells were passed through a 100-mesh nylon mesh sieve and centrifuged at $400 \mathrm{xg}$ for $8 \mathrm{~min}$ at room temperature. The cells were resuspended in Dulbecco's modified Eagle's medium (DMEM)/Ham's F12 (Gibco; Thermo Fisher Scientific, Inc., Waltham, MA, USA). The isolated chondrocytes $\left(1 \times 10^{5}\right.$ cells $\left./ \mathrm{ml}\right)$ in culture flasks were counted and cultured at $37^{\circ} \mathrm{C}$. The medium was refreshed every $48 \mathrm{~h}$.

The SW-1353 human bone chondrosarcoma cell line was obtained from the Institute of Biochemistry and Cell Biochemistry and Cell Biology (Shanghai, China). Cells were cultured in DMEM with $10 \%$ fetal bovine serum (FBS; Gibco; Thermo Fisher Scientific, Inc.) and incubated at $37^{\circ} \mathrm{C}$ for $24 \mathrm{~h}$ before transferring onto 96 -well plates at $9 \times 10^{3}$ cells/well.

Cell counting kit-8 (CCK-8) assay. Cell viability was assessed using a CCK-8 assay (Dojindo Molecular Technologies, Inc., Kumamoto, Japan) assay. Briefly, cells were seeded onto 96-well plates at a density of 7,000 cells/well and incubated at $37^{\circ} \mathrm{C}$ for $24 \mathrm{~h}$. Following transfection, CCK-8 solution was added to each well prior to incubation for $1 \mathrm{~h}$ at $37^{\circ} \mathrm{C}$ in the dark. The optical density values were determined at dual wavelengths of 450 and $630 \mathrm{~nm}$ (reference wavelength) to ascertain cell viability using a microplate reader (Thermo Labsystems, Santa Rosa, CA, USA).

Plasmid construction and transfection of miRNA. Human chondrocytes and SW-1353 cells were transfected with the miR-204-5p mimic (uucccuuugucauccuaugccu; Ambion; Thermo Fisher Scientific, Inc.) or antisense inhibitor anti-miR-204 (aggcauag gaugacaaagggaa; Ambion; Thermo Fisher Scientific, Inc.) at a concentration of $50 \mathrm{nM}$ using Lipofectamine ${ }^{\circledR} 2000$ transfection reagent (Invitrogen; Thermo Fisher Scientific, Inc.). For the control, cells were transfected with Lipofectamine ${ }^{\circledR}$ RNAiMAX (Invitrogen; Thermo Fisher Scientific, Inc.) with a scrambled control miRNA.

The reporter plasmid was constructed by inserting Runx2 mRNA 3'-UTRs downstream of the firefly luciferase gene in the pmirGLO vector (Promega Corporation, Madison, WI, USA). Cells were seeded onto plates, incubated at $37^{\circ} \mathrm{C}$ and were harvested when the cell monolayer reached $70 \%$ confluence. Recombinant plasmid (200 ng) and miR-204-5p mimic (200 ng) were co-transfected into the cells. Plasmid suspension and Lipofectamine 2000 reagent were incubated in FBS-free DMEM for $20 \mathrm{~min}$ and then added to 48 -well plates. After $6 \mathrm{~h}$ of incubation, DMEM was added to the wells.

Luciferase gene reporter assay. Primary rat chondrocytes were harvested and subjected to luciferase activity assays as described previously (17). Briefly, after $48 \mathrm{~h}$ of incubation, growth medium was removed and cells were washed with PBS twice. Cells were broken down by adding $100 \mu \mathrm{l}$ passive lysis buffer (Guangzhou RiboBio Co., Ltd., Guangzhou, China) to each well and placed on ice for $30 \mathrm{~min}$. Cell lysates were transferred to 96-well microplates. Dual Luciferase assay kits (Promega Corporation) were used according to the manufacturer's protocol and luciferase activity was determined using a microplate reader at $540 \mathrm{~nm}$. An equal volume of Dual-Glo ${ }^{\circledR}$ Stop\&Glo Reagent (Promega Corporation) was added to the wells, and Renilla luminescence was determined 
$48 \mathrm{~h}$ after transfection using the dual-luciferase reporter assay system (Promega Corporation). Rat Runx2 3'-UTR (NM_001278483.1, positions 5,251-5,575) was cloned into the psiCHECK ${ }^{\mathrm{TM}}-2$ vector (Guangzhou RiboBio Co., Ltd.), and the primers used to construct the plasmid were: Forward, 5'-ccgCTCGAGtaacgtacagtaatttctta-3' (capitals denote the XhoI site) and reverse, 5'-ataagaatGCGGCCGCtcatgagggga gaaaatgcc-3' (capitals denote the NotI site). The experiments were performed in triplicate.

Immunohistochemistry (IHC) and Safranin O/Fast Green staining. Tissue specimens were fixed in $10 \%$ formaldehyde at $4^{\circ} \mathrm{C}$ for 7 days, decalcified in $4.13 \%$ EDTA at $4^{\circ} \mathrm{C}$ for 14 days and then embedded in paraffin. Joints were sliced into $5 \mu \mathrm{m}$ continuous sections and stained with Safranin O/Fast Green and antibody against Runx2 (cat. no. ab2398; 1:200; Abcam, Cambridge, UK). Histomorphometric analysis was performed with quantification of the Safranin O-positive cartilage area and its thickness, and Runx2-positive cells using Pro Plus software (version 6.0; Media Cybernetics, Inc., Rockville, MD, USA). OA scoring was analyzed using a modified Mankin's Score system $(18,19)$.

Reverse transcription-quantitative polymerase chain reaction (RT-qPCR). Cartilages of the femoral head and knee joint were obtained from patients with OA. Control articular cartilages were obtained from patients with idiopathic osteonecrosis of the femoral condyle. Cartilage from normal and OA donors were milled to a fine powder in liquid nitrogen. Total RNAs and miRNA were extracted from tissue samples obtained from patients and rats with $\mathrm{OA}$ and from cell cultures using TRIzol ${ }^{\circledR}$ (Thermo Fisher Scientific, Inc.). miRNA was purified using a mirVana isolation kit (Ambion; Thermo Fisher Scientific, Inc.), according to the manufacturer's protocol. The purity of total RNAs and miRNA was determined using a NanoDrop instrument (Thermo Fisher Scientific, Inc.). For detecting the expression level of Runx 2 mRNA, the cDNA was prepared from $2 \mu \mathrm{g}$ total RNA using a cDNA synthesis kit (Bio-Rad Laboratories, Inc., Hercules, CA, USA). Expression of mature miRNA was quantified using a TaqMan miRNA assay kit (Applied Biosystems; Thermo Fisher Scientific, Inc.). Purified miRNA was reverse-transcribed using a TaqMan miRNA reverse transcription kit (Applied Biosystems; Thermo Fisher Scientific, Inc.). Expression of the mRNA level was quantified by adding $1 \mu \mathrm{g}$ synthesized first-strand cDNA in a $20-\mu 1$ reaction mixture using the PrimeScript RT reagent kit (Takara Biotechnology Co., Ltd., Dalian, China). qPCR was performed using a PCR instrument (7300 Step One Plus; Applied Biosystems; Thermo Fisher Scientific, Inc.). Thermocycling conditions were as follows: 40 cycles of $95^{\circ} \mathrm{C}$ for $5 \mathrm{sec}$ and $60^{\circ} \mathrm{C}$ for $34 \mathrm{sec}$. As an internal control, U6 primers (forward, 5'-CTCGCTTCGGCAGCACA-3' and reverse, 5'-AACGCTTCACGAATTTGCGT-3') were used for RNA template normalization. The relative expression of each target gene was calculated using the $2^{-\Delta \Delta \mathrm{Cq}}$ method (20). The stem-loop primer for human miR-204-5p was 5'-ACACTC CAGCTGGGTTCCCTTTGTCATCCTAT-3', and the reverse primer was 5'-CTCAACTGGTGTCGTGGA-3'. Primers for rat Runx2 were 5'-GTTATGAAAAACCAAGTAGCCAGG T-3' (forward) and 5'-GTAATCTGACTCTGTCCTTGTGGA
T-3' (reverse). Primers for human Runx2 were 5'-TCTCTG ACCGCCTCAGTGAT-3' (forward) and 5'-CAGAGGTGG CAGTGTCATCAT-3' (reverse).

Western blotting. Cartilages of the femoral head and knee joint were obtained from patients with OA. Control articular cartilages were obtained from patients with idiopathic osteonecrosis of the femoral condyle. Total protein was extracted from each sample using radioimmunoprecipitation assay lysis buffer (Beyotime Institute of Biotechnology, Haimen, China) and the protein concentrations were assessed using a bicinchoninic acid protein assay kit (Beyotime Institute of Biotechnology). Equal amounts of protein were loaded and separated discontinuously by SDS-PAGE (12\% gels), and subsequently transferred onto a polyvinylidene fluoride membrane (GE Healthcare Life Sciences, Little Chalfont, UK). Membranes were blocked for $1 \mathrm{~h}$ at room temperature with 5\% non-fat milk in Tris-buffered saline plus $0.5 \%$ Tween-20 (TBST). The membranes were incubated with primary antibodies, including anti-Runx2 (cat. no. ab76956), anti-collagen II (cat. no. ab34712), anti-collagen X (cat. no. ab49945), anti-MMP-1 (cat. no. ab52631), anti-MMP-13 (cat.no. ab39012), and anti-COMP (cat.no. ab74524) (all Abcam) overnight at $4^{\circ} \mathrm{C}$. Antibodies were diluted with TBST at ratio of 1:1,000. The membrane was washed several times and incubated with horseradish peroxidase-conjugated anti-rabbit secondary antibodies (1:200; cat. no. ab7090; Abcam) for 2 h. Following washing, blots were visualized using enhanced chemiluminescence reagent (Forevergen Biosciences, Guangzhou, China). Signals were densitometrically assessed with the gel analysis tool of ImageJ software (version $1.48 \mathrm{v}$; National Institutes of Health, Bethesda, MD, USA).

Statistical analysis. For all analyses, the measurements obtained from the groups are expressed as the mean \pm standard deviation. Analysis of variance followed by Tukey's test was performed for multiple groups and Student's t-test was applied to analyze the differences between two groups. $\mathrm{P}<0.05$ was considered to indicate a statistically significant difference.

\section{Results}

Runx2 is induced in human OA tissues where miR-204-5p is downregulated. Relative mRNA expression of Runx 2 was significantly increased in OA tissues compared with necrotic bone tissues ( $\mathrm{P}<0.001$; Fig. 1A). Similarly, the protein expression of Runx 2 was increased in OA tissues compared with necrotic bone tissues (Fig. 1B), which was revealed to be significant $(\mathrm{P}<0.01$; Fig. 1C). In contrast, the relative level of miR-204-5p was significantly decreased in OA tissue samples compared with that in bone necrosis samples ( $\mathrm{P}<0.001$; Fig. 1D).

Overexpression of miR-204-5p downregulates Runx2 in rat primary chondrocytes and the $S W-1353$ human bone chondrosarcoma cell line. As presented in Fig. 2A, the relative level of miR-204-5p was significantly increased in cells transfected with miR-204-5p mimic and this expression was suppressed when its inhibitor was transfected. These results may be indicative of successful transfection; therefore, the mimic and inhibitor were qualified for follow-up examination. The relative mRNA level of Runx 2 was significantly decreased 
in the presence of miR-204-5p mimic compared with its NC $(\mathrm{P}<0.05)$, and a significant increase was detected in the presence of miR-204-5p inhibitor compared with miR-204-5p mimic $(\mathrm{P}<0.05$; Fig. $2 \mathrm{~B})$. A similar expression pattern was also observed for Runx2 protein detected by western blot analysis (Fig. 2C and D).

Overexpression of miR-204-5p inhibits chondrocyte proliferation. Cell proliferation was significantly suppressed when miR-204-5p mimic was transfected, compared with $\mathrm{NC}$, and this decrease in cell proliferation was reversed following transfection of miR-204-5p inhibitor $(\mathrm{P}<0.05)$ in rat primary chondrocytes (Fig. 3A) and the SW-1353 cell line (Fig. 3B).

miR-204-5p regulates the expression level of OA-associated molecules and Runx2 is a direct target of miR-204-5p. Protein expression of collagen II was increased, whereas that of collagen X, matrix metalloproteinase (MMP)-1 and MMP-13 was decreased in miR-204-5p mimic-transfected cells compared with $\mathrm{NC}$ in rat primary chondrocytes and the SW-1353 cell line (Fig. 4A). These differences were revealed to be significant $(\mathrm{P}<0.05$; Fig. $4 \mathrm{~B}$ and $\mathrm{C})$. These alterations in the expression level of OA-associated mediators caused by miR-204-5p mimic were reversed by miR-204-5p inhibitor (Fig. 4A-C). In addition, a putative miR-204-5p-binding site for Runx 2 mRNA is presented in Fig. 4D. Relative luciferase activity was significantly decreased in the wild-type following transfection with miR-204-5p mimic, compared with NC $(\mathrm{P}<0.05)$. Such events were not observed in cells transfected with empty vector and/or mutant 3'-UTR-binding site of Runx2 mRNA ( $P>0.05$; Fig. 4E).

miR-204-5p agomir attenuates the loss of articular cartilage in rats. Articular cartilage tissues of femoral condyle were stained with Safranin O/Fast Green and the cartilage was stained red, whereas the subchondral bone appeared blue. Following treatment for 10 weeks (Fig. 5A), the rats in the sham group exhibited uniform staining with Safranin O, indicating the complete structure of joint cartilage. Rats in the OA group exhibited features of $\mathrm{OA}$, including surface undulations, a marked decrease in Safranin O-positivity on the superficial layer, and appearance of hypertrophic chondrocytes at the deeper layer. After 15 weeks (Fig. 5B), these histological manifestations were more pronounced. Osteophytes (Runx2-positive) were observable in certain sections of articular cartilage in the OA group, but scarcely observed in the agomir group (data not shown). Histomophometric analysis revealed that the thickness of cartilage was significantly decreased in the OA group $(\mathrm{P}<0.05$; Fig. $5 \mathrm{C})$. In addition, Mankin's score was significantly increased in the OA group compared with the sham group $(\mathrm{P}<0.05$; Fig. 5D). However, miR-204-5p agomir significantly inhibited the loss of articular cartilage and decreased Mankin's score, indicating that cartilage degeneration was suppressed by miR-204-5p agomir in rats with OA. IHC analysis identified that Runx2 presented at superficial layer where there was a significant decrease in Safranin O staining. As presented in the IHC images, positive staining of Runx 2 was located in nuclei of chondrocytes. After 10 and 15 weeks of treatment, the numbers of Runx2-positive chondrocytes were increased in the OA group compared with
A
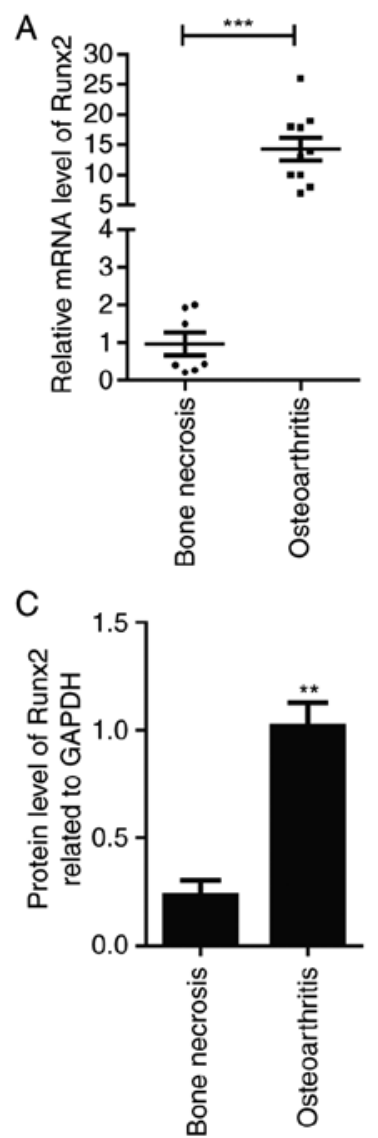

B

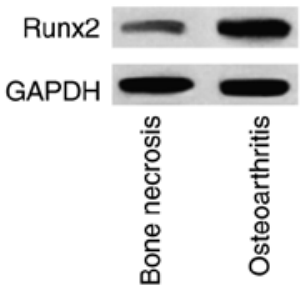

Figure 1. Runx 2 and miR-204-5p expression in human OA tissues. (A) Relative mRNA expression level of Runx2 in tissue specimens. (B) Relative expression of miR-204-5p in tissue specimens. (C) Protein expression of Runx2 in tissue specimens. (D) Quantification of protein expression. ${ }^{* *} \mathrm{P}<0.01$ ${ }^{* * *} \mathrm{P}<0.001$ vs. bone necrosis group. Runx 2 , Runt-related transcription factor- 2 miR, microRNA

in the Sham group, whereas that in the agomir treatment group was notably decreased compared with the OA group.

miR-204-5p agomir alters the expression of OA-associated mediators in rats. At 1 day, 5 weeks, 10 weeks and 15 weeks after miR-204-5p agomir injection, relative expression levels of miR-204-5p and Runx2 were determined by western blotting. Except for no significant alteration between the sham and OA groups at 1 day, the miR-204-5p level was significantly decreased in OA group (compared with the sham group) and significantly increased in the OA+agomir group (compared with the OA group) at different time points $(\mathrm{P}<0.05$; Fig. 6A). The Runx 2 protein level at different time points exhibited the same expression pattern with a considerably increased level in OA group and decreased level in the $\mathrm{OA}+$ agomir group, and the Runx 2 expression level increased with the treatment duration (Fig. 6B), which was revealed to be significant (Fig. 6C). After 10 weeks, protein expression of MMP-1, MMP-13, cartilage oligomeric matrix protein (COMP) fragment and collagen $\mathrm{X}$ was notably increased, whereas the expression level of collagen II and aggrecan was decreased in the OA group compared with the sham group (Fig. 6D). These alterations in OA were effectively reversed with miR-204-5p agomir treatment (Fig. 6D). Differences in the expression levels of the various proteins 

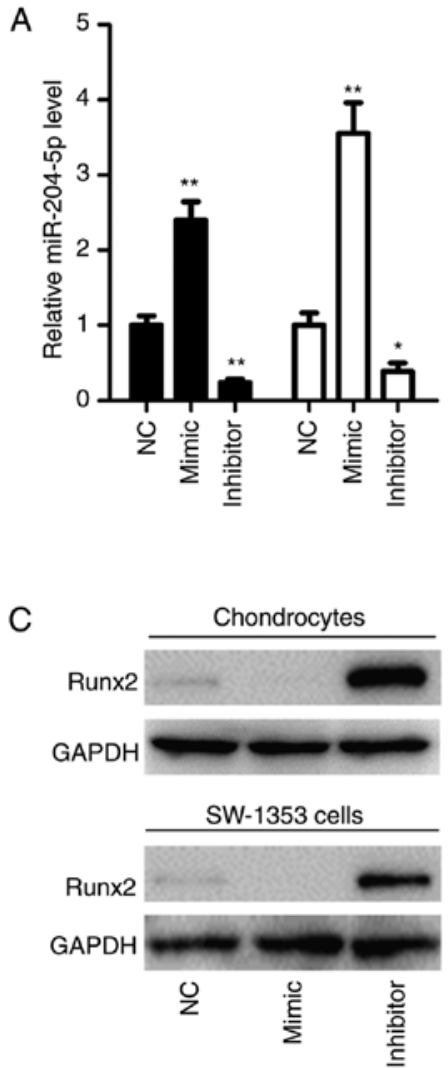

B
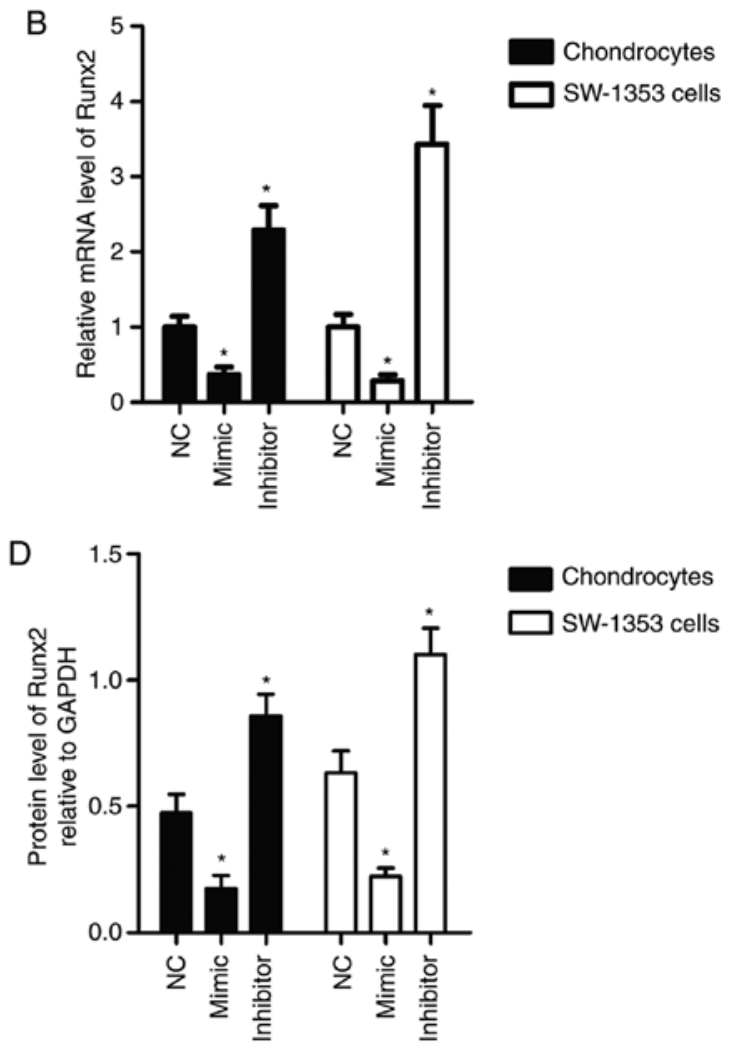

Figure 2. Runx2 and miR-204-5p expression in transfected cells. (A) Relative miR-204-5p expression in primary chondrocytes and SW-1353 cells. (B) Relative mRNA expression of Runx2 in primary chondrocytes and SW-1353 cells. (C) Protein expression of Runx2 in primary chondrocytes and SW-1353 cells. (D) Quantification of protein expression. " $\mathrm{P}<0.05,{ }^{* * *} \mathrm{P}<0.01 \mathrm{vs}$. NC group. Runx2, Runt-related transcription factor-2; miR, microRNA; NC, negative control.

were significantly different between the treatment groups $(\mathrm{P}<0.05$; Fig. 6E).

\section{Discussion}

In the present study, it was identified that miR-204-5p was downregulated in cartilage tissue of patients with OA where Runx2 was overexpressed. This negative-association expression pattern was also observed in SW-1353 human bone chondrosarcoma cells and rat primary chondrocytes. In order to elucidate the function of miR-204-5p, cell proliferation was determined in the two types of cell, and each cell type was divided into three different subgroups: miR-204-5p mimic, miR-204-5p inhibitor and NC. It was identified that overexpressed miR-204-5p significantly inhibited the cell proliferation in the two cell types. The well-established markers collagens II, X, MMP-1 and MMP-13 were investigated for the hypertrophic stage of chondrocyte differentiation and miR-204-5p-binding activity to Runx2. The results identified that the expression levels of these markers were significantly increased with miR-204-5p overexpression and that Runx2 was a target of miR-204-5p. At 10 and 15 weeks after the treatment, the severity of OA-like phenotype was analyzed by grading histological sections using Mankin's score system by two blinded observers in addition to examination of Runx 2 protein expression and cartilage thickness. Following miR-204-5p agomir treatment, cartilage thickness was increased, whereas Mankin's score was decreased, and the number of Runx2-positive cells was also decreased. In order
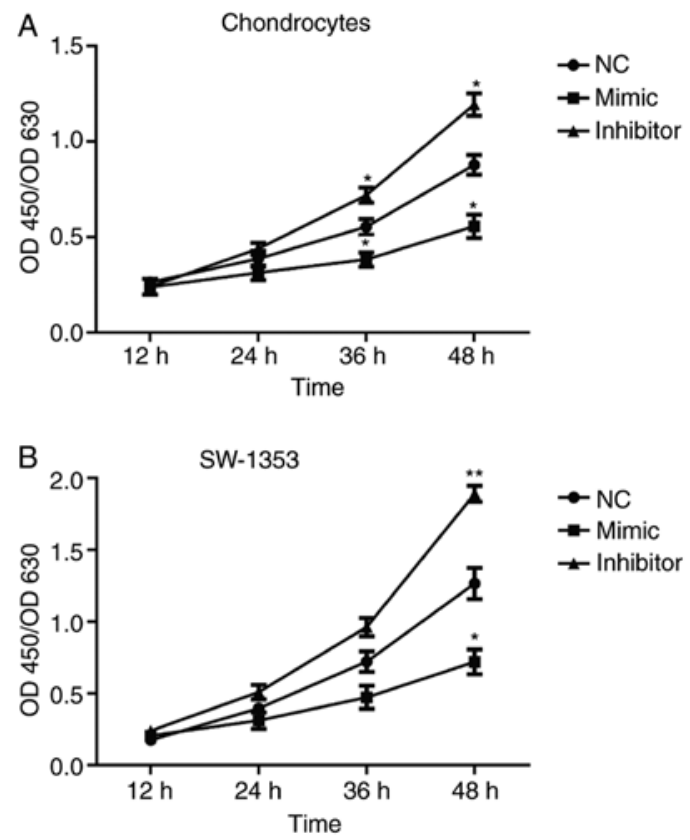

Figure 3. Inhibition of cell proliferation is induced by overexpression of miR-204-5p. Proliferation status in cells transfected with miR-204-5p mimic, miR-204-5p inhibitor and NC in (A) primary chondrocytes and (B) SW-1353 cells. ${ }^{*} \mathrm{P}<0.05,{ }^{* *} \mathrm{P}<0.01$ vs. $\mathrm{NC}$ group. Runx 2 , Runt-related transcription factor-2; miR, microRNA; NC, negative control; OD, optical density.

to elucidate further the potential function of miR-204-5p in age-associated OA, the expression levels of miR-204-5p and 

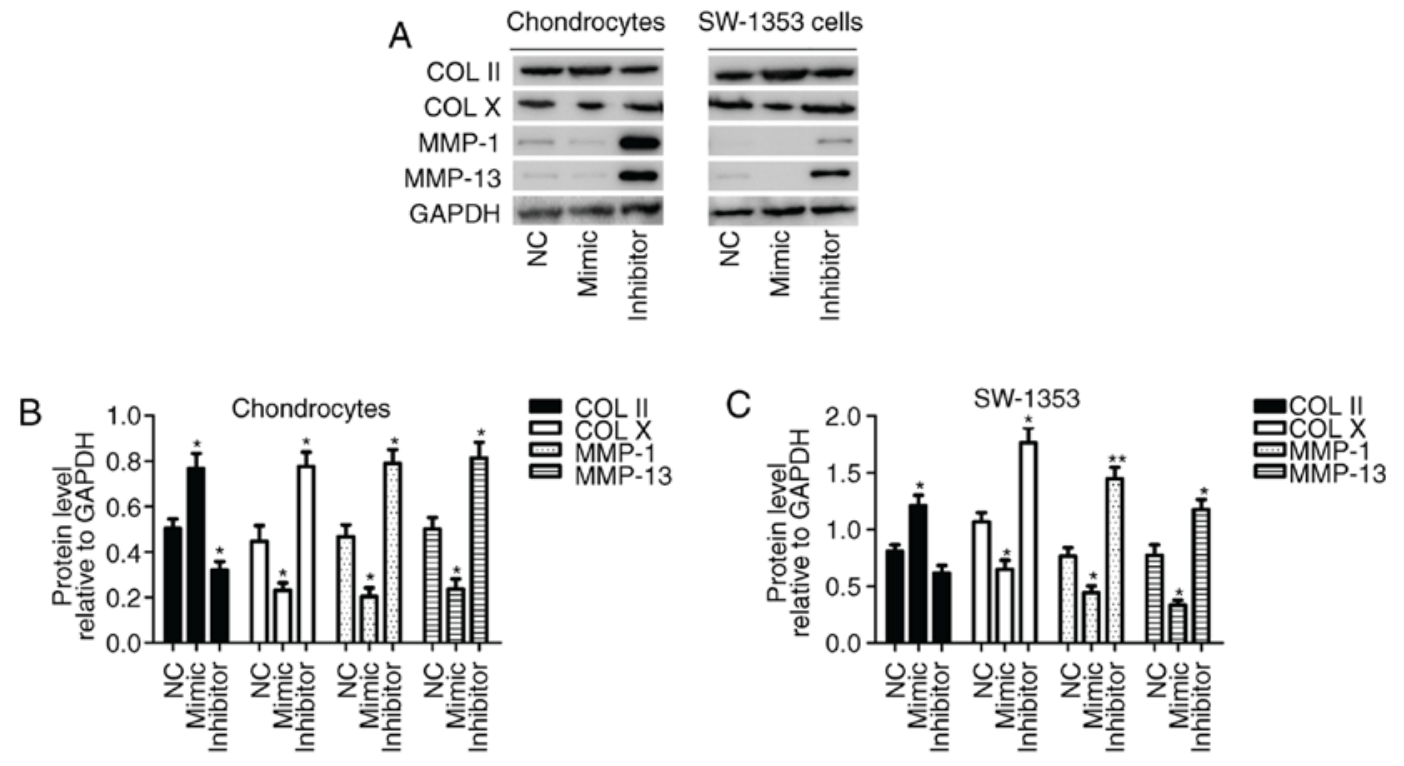

$\mathrm{D}$

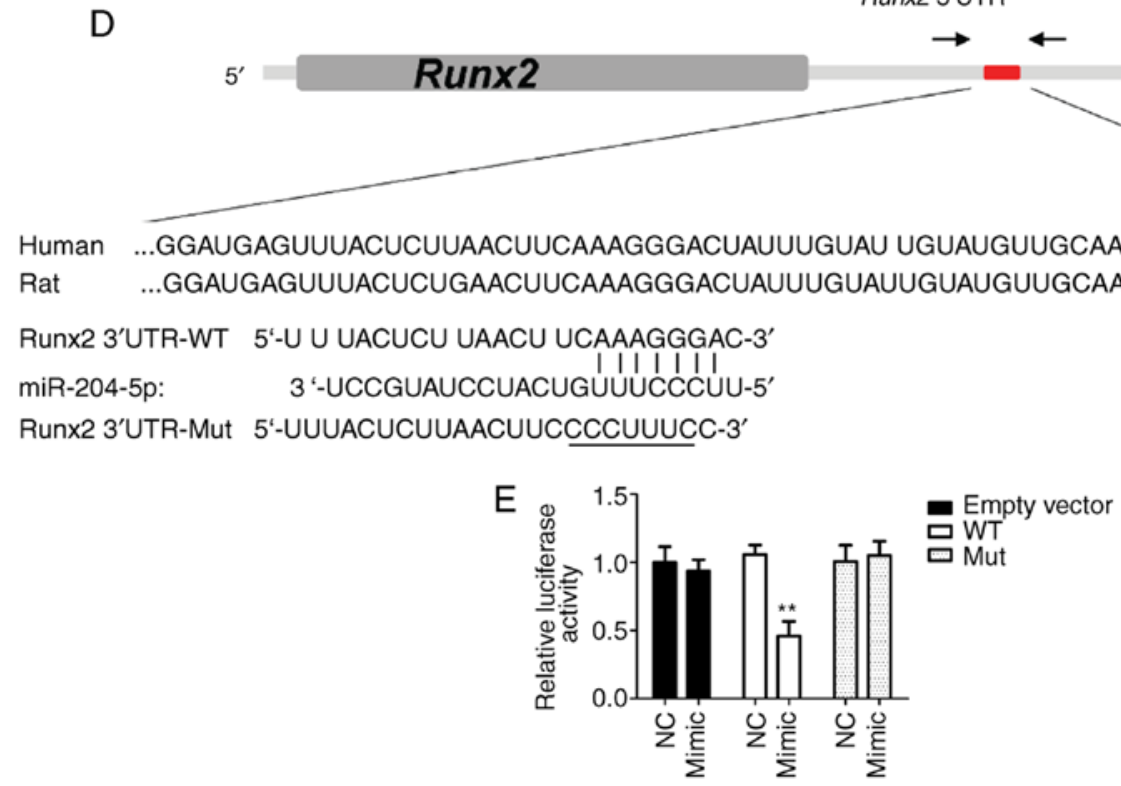

Figure 4. Protein expression levels of OA-associated molecules and luciferase activity in transfected cells. (A) Image of immunoblot by western blotting. Quantification of COL II and X, MMP-1 and MMP-13 expression in (B) primary chondrocytes and (C) SW-1353 cells. (D) Putative miR-204-5p target sequences in the Runx2 3'-UTR. (E) Relative luciferase activity in primary chondrocytes and SW-1353 cells transfected with WT and Mut forms of target sequences. "P $<0.05,{ }^{* *} \mathrm{P}<0.01$ vs. NC group. Runx2, Runt-related transcription factor-2; miR, microRNA; OA, osteoarthritis; COL, collagen; MMP, matrix metalloproteinase; UTR, untranslated region; WT, wild-type; Mut, mutant.

Runx2 were determined at different time points during the treatment. The agomir treatment group exhibited a significant increase in miR-204-5p expression, but a decrease in Runx2 expression. Following agomir treatment for 10 weeks, osteophyte formation was evaluated by examining the associated molecules, and a decrease in MMP-1, MMP-13, COMP fragment, collagen $\mathrm{X}$, and an increase in collagen II and aggrecan were identified.

How specific miRNAs influence cartilage hemostasis and the mechanism of OA development has been the focus of previous studies (21-23). As a tumor suppressor, miR-204-5p has been intensively investigated in different diseases (24). Numerous miRNAs appear to be important modulators of chondrogenesis and OA. Their expression is frequently altered in $\mathrm{OA}$, and a number are functionally implicated in the pathogenesis of the disease (25). However, the function of miR-204-5p in OA and the underlying molecular mechanism involved has not yet been investigated.

During bone development, the balance between chondrocyte proliferation and differentiation is crucial for maintaining normal bone growth and endochondral bone formation. Disturbed chondrocyte proliferation as well as hypertrophic differentiation leads to alterations in bone structure and stability (26). Runx2 serves an important function in the maturation of chondrocytes, which is a prerequisite for endochondral ossification and this is an essential process for the development of OA (27). Runx2 also serves an essential function in the differentiation of mesenchymal progenitor cells into osteoblasts, and its deficiency leads to a lack of endochondral and intramembranous bone formation because of the arrest 


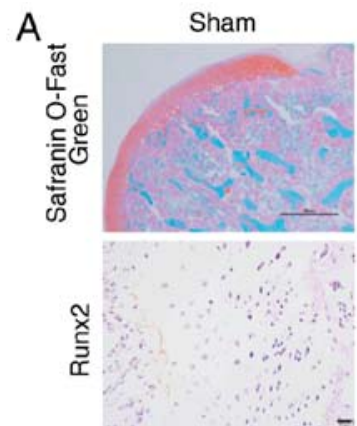

$\mathrm{B}$

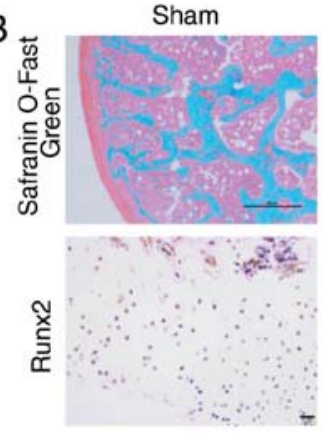

$\mathrm{OA}$

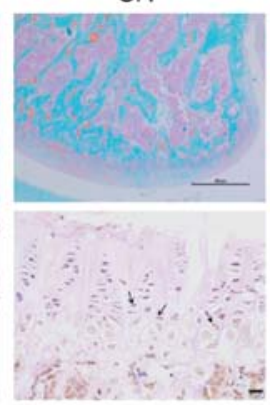

$\mathrm{OA}$

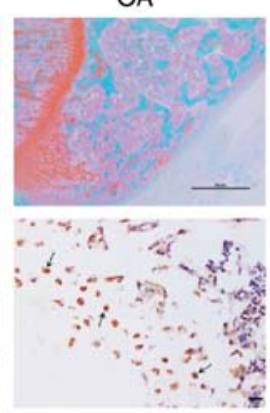

$\mathrm{OA}+$ Agomir

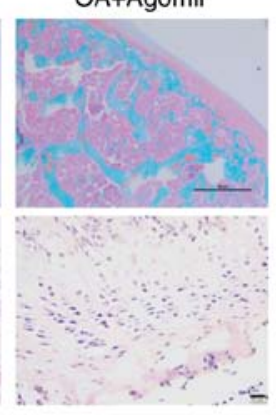

$\mathrm{OA}+\mathrm{Agomir}$

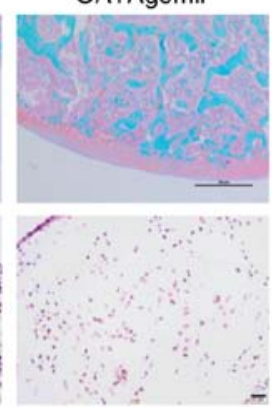

C

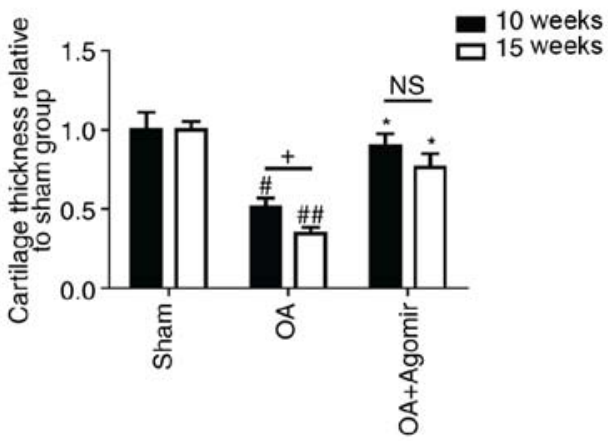

D

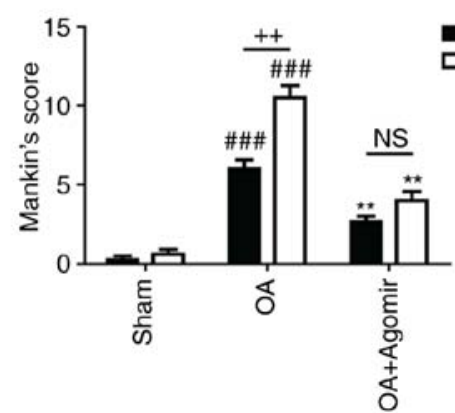

Figure 5. Representative histological staining of articular cartilage of femoral of rats from sham, OA and OA+agomir treatment groups. Safranin O/Fast Green staining (scale bar, $100 \mu \mathrm{m}$ ) and Runx2-positive staining (arrow) with immunohistochemistry (scale bar, $20 \mu \mathrm{m}$ ) at (A) 10 weeks and (B) 15 weeks after treatment (magnification, $\mathrm{x} 400$ ). (C) Assessment of relative cartilage thickness and (D) Mankin's score at 10 weeks and 15 weeks after treatment. ${ }^{\#} \mathrm{P}<0.05$, ${ }^{\# \#} \mathrm{P}<0.01,{ }^{\# \# \#} \mathrm{P}<0.001$ vs. the sham group; ${ }^{*} \mathrm{P}<0.05,{ }^{* *} \mathrm{P}<0.01$ vs. the $\mathrm{OA}$ group; ${ }^{+} \mathrm{P}<0.05,{ }^{+} \mathrm{P}<0.01$. OA, osteoarthritis; Runx 2 , Runt-related transcription factor-2; NS, not significant.
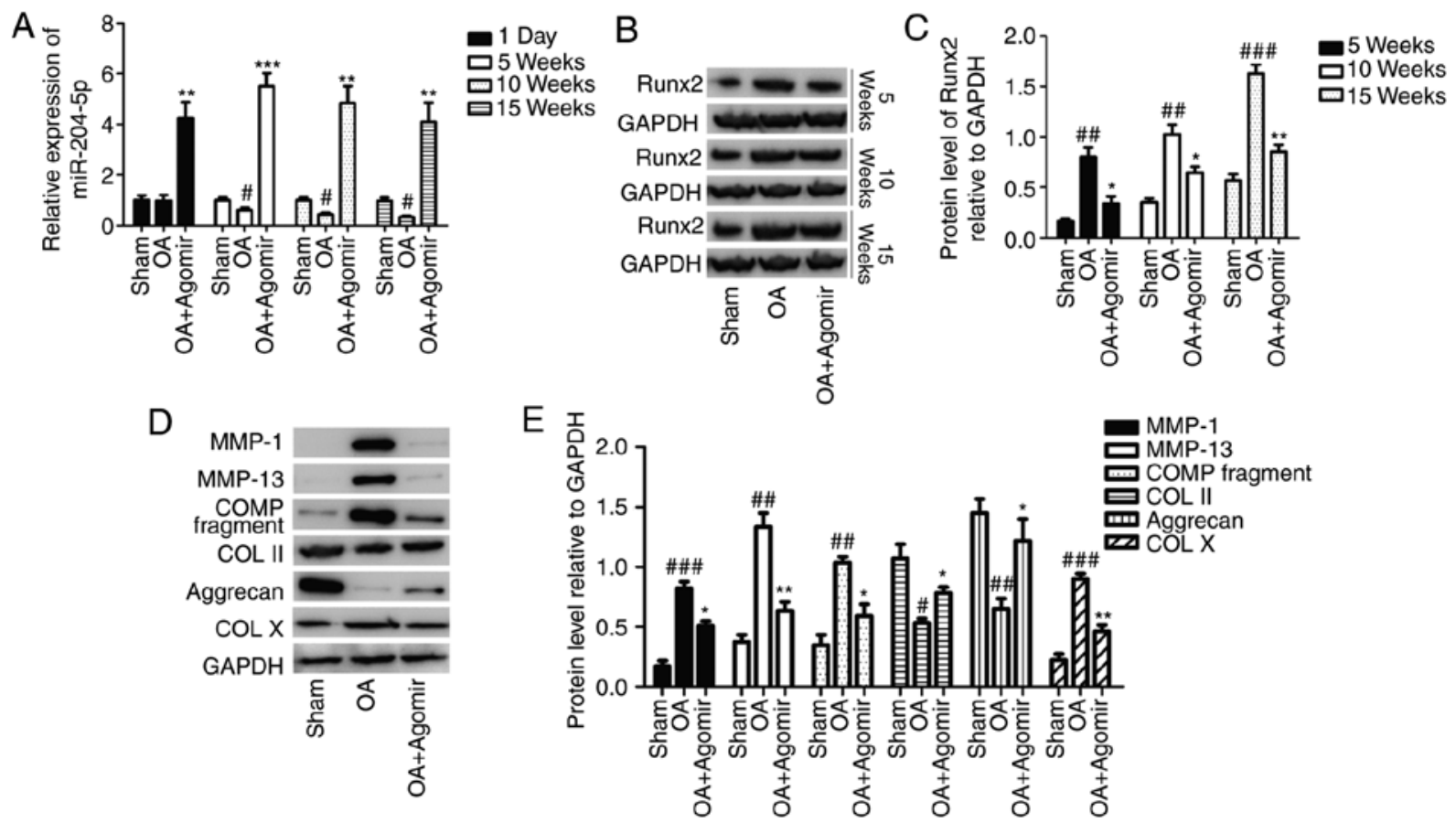

Figure 6. Expression of Runx2 and OA-associated molecules at 1 day, 5 weeks, 10 weeks and 15 weeks after treatment. (A) Relative expression of miR-204-5p at different time points. (B) Runx2 protein expression at different time points. (C) Quantification of protein expression. (D) Protein expression level of COL II and X, COMP fragment, aggrecan, MMP-1 and MMP-13 at 10 weeks after treatment. (E) Quantification of protein expression. ${ }^{\#} \mathrm{P}<0.05$, ${ }^{\# \#} \mathrm{P}<0.01,{ }^{\# \# "} \mathrm{P}<0.001$ vs. the sham group; ${ }^{*} \mathrm{P}<0.05,{ }^{* *} \mathrm{P}<0.01$ vs. the OA group. Runx2, Runt-related transcription factor-2; OA, osteoarthritis; miR, microRNA; COL, collagen; COMP, cartilage oligomeric matrix protein; MMP, matrix metalloproteinase.

of osteoblast differentiation $(28,29)$. Runx2 expression was consistently high at all stages of chondrocyte differentiation (30). No hypertrophic cells were detected in Runx2/Runx3 double mutants. In contrast, overexpression of Runx 2 is sufficient to initiate hypertrophic differentiation (31). The results of the present study indicated that rats with surgically induced 
OA exhibited an OA-like phenotype as well as increased expression of Runx2 protein adjacent to the area with cartilage degradation. With miR-204-5p treatment, the severity of disease and OA degradation was ameliorated, and the Runx2 expression was decreased. In addition, it was also identified that the production of Runx 2 was gradually increased as the rats aged. Therefore, we hypothesized that Runx2 may also be involved in age-associated OA (32).

In each phase of endochondral ossification, cells were subjected to consistent alteration in shapes and arrangement (2). Subsequent to undifferentiated mesenchymal progenitor cells differentiating into chondrocytes, cells commence the production of typical cartilage components, including collagens II, IX and XI, and the proteoglycan aggrecan (33). During this stage, chondrocytes maintain a low proliferation rate. Following entry of chondrocytes into the proliferation stage with several rounds of division, the cells arrange into longitudinal columns and begin to express collagen VI (34). Further differentiation into hypertrophic chondrocytes induces the production of collagen X. Finally, hypertrophic chondrocytes decrease the production of collagens II, IX and XI, and MMP-13 and transcription factor Runx2 (35). Among them, MMP-13 and collagen $\mathrm{X}$ are well-established markers for late chondrocyte differentiation. COMP is another important regulator in the maintenance of collagens I and II networks (36). In the present study, the expression levels of all these mediators were determined in order to elucidate the molecular mechanism accompanied by chondrocyte proliferation in OA development, and the results confirmed the anti-OA effect of miR-204-5p in the rat OA models.

In conclusion, the results of the present study identified that, by targeting Runx 2 , miR-204-5p decreased the chondrocyte proliferation and ameliorated the OA-like phenotype in rats with surgically induced OA, and suggested that miR-204-5p may be investigated as a novel therapeutic agent for the treatment of OA.

\section{Acknowledgements}

Not applicable.

\section{Funding}

No funding was received.

\section{Availability of data and materials}

All data generated or analyzed during this study are included in this published article.

\section{Authors' contributions}

XLL conceived and designed the study. JQC and XYH performed experiments and were major contributors in writing the manuscript. XQ and XYJ made significant contributions to acquisition and analysis of data. JQC and XQ performed the statistical analysis. JQC and XYH were responsible for biological samples collection. XLL revised the paper critically for important intellectual content. All authors read and approved the final manuscript.

\section{Ethics approval and consent to participate}

All human studies were approved by the institutional ethics review board of The Sixth People's Hospital Affiliated to Shanghai Jiao Tong University (Shanghai, China). Written informed consent was obtained from each patient. All animal procedures were approved by the Animal Care and Use Committee of The Sixth People's Hospital Affiliated to Shanghai Jiao Tong University.

\section{Patient consent for publication}

Not applicable.

\section{Competing interests}

The authors declare that they have no competing interests.

\section{References}

1. Kim HA, Lee YJ, Seong SC, Choe KW and Song YW: Apoptotic chondrocyte death in human osteoarthritis. J Rheumatol 27: 455-462, 2000.

2. Dreier R: Hypertrophic differentiation of chondrocytes in osteoarthritis: The developmental aspect of degenerative joint disorders. Arthritis Res Ther 12: 216, 2010.

3. ChenH,Ghori-JavedFY,RashidH,AdhamiMD,SerraR,GutierrezSE and Javed A: Runx2 regulates endochondral ossification through control of chondrocyte proliferation and differentiation. JBone Miner Res 29: 2653-2665, 2014.

4. Yoshida CA, Yamamoto H, Fujita T, Furuichi T, Ito K, Inoue K, Yamana K, Zanma A, Takada K, Ito Y and Komori T: Runx2 and Runx 3 are essential for chondrocyte maturation, and Runx2 regulates limb growth through induction of Indian hedgehog. Genes Dev 18: 952-963, 2004.

5. Ji Q, Xu X, Xu Y, Fan Z, Kang L, Li L, Liang Y, Guo J, Hong T, Li Z, et al: miR-105/Runx2 axis mediates FGF2-induced ADAMTS expression in osteoarthritis cartilage. J Mol Med 94: 681-694, 2016.

6. Altorok N, Tsou PS, Coit P, Khanna D and Sawalha AH: Genome-wide DNA methylation analysis in dermal fibroblasts from patients with diffuse and limited systemic sclerosis reveals common and subset-specific DNA methylation aberrancies. Ann Rheum Dis 74: 1612-1620, 2015.

7. Guo F, Han X, Wu Z, Cheng Z, Hu Q, Zhao Y, Wang Y and Liu C: ATF6a, a Runx2-activable transcription factor, is a new regulator of chondrocyte hypertrophy. J Cell Sci 129: 717-728, 2016.

8. Yates LA, Norbury CJ and Gilbert RJ: The long and short of microRNA. Cell 153: 516-519, 2013.

9. Le LT, Swingler TE and Clark IM: Review: The role of microRNAs in osteoarthritis and chondrogenesis. Arthritis Rheum 65: 1963-1974, 2013.

10. Yu C, Li L, Xie F, Guo S, Liu F, Dong N and Wang Y: LncRNA TUG1 sponges miR-204-5p to promote osteoblast differentiation through upregulating Runx2 in aortic valve calcification. Cardiovasc Res 114: 168-179, 2018.

11. Shang G, Wang Y, Xu Y,Zhang S, Sun X, Guan H, Zhao X, Wang Y, Li Y and Zhao G: Long non-coding RNA TCONS_00041960 enhances osteogenesis and inhibits adipogenesis of rat bone marrow mesenchymal stem cell by targeting miR-204-5p and miR-125a-3p. J Cell Physiol 233: 6041-6051, 2018.

12. Huang J, Zhao L, Xing L and Chen D: MicroRNA-204 regulates Runx 2 protein expression and mesenchymal progenitor cell differentiation. Stem Cells 28: 357-364, 2010.

13. Zhang Y, Xie RL, Croce CM, Stein JL, Lian JB, van Wijnen AJ and Stein GS: A program of microRNAs controls osteogenic lineage progression by targeting transcription factor Runx 2 . Proc Natl Acad Sci USA 108: 9863-9868, 2011.

14. Belaya Z, Grebennikova T, Melnichenko G, Nikitin A, Solodovnikov A, Brovkina O, Grigoriev A, Rozhinskaya L, Lutsenko A and Dedov I: Effects of active acromegaly on bone mRNA and microRNA expression patterns. Eur J Endocrinol 178: 353-364, 2018 
15. Rogart JN, Barrach HJ and Chichester CO: Articular collagen degradation in the Hulth-Telhag model of osteoarthritis. Osteoarthritis Cartilage 7: 539-547, 1999.

16. Chen L, Li Q, Wang J, Jin S, Zheng H, Lin J, He F, Zhang H, Ma S, Mei J and Yu J: MiR-29b-3p promotes chondrocyte apoptosis and facilitates the occurrence and development of osteoarthritis by targeting PGRN. J Cell Mol Med 21: 3347-3359, 2017.

17. Jin $Y$, Chen Z, Liu X and Zhou X: Evaluating the microRNA targeting sites by luciferase reporter gene assay. Methods Mol Biol 936: 117-127, 2013

18. Kuroki H, Nakagawa Y, Mori K, Ohba M, Suzuki T, Mizuno Y, Ando K, Takenaka M, Ikeuchi K and Nakamura T: Acoustic stiffness and change in plug cartilage over time after autologous osteochondral grafting: Correlation between ultrasound signal intensity and histological score in a rabbit model. Arthritis Res Ther 6: R492-R504, 2004.

19. Bulstra SK, Buurman WA, Walenkamp GH and Van der Linden AJ: Metabolic characteristics of in vitro cultured human chondrocytes in relation to the histopathologic grade of osteoarthritis. Clin Orthop Relat Res: 294-302, 1989.

20. Livak KJ and Schmittgen TD: Analysis of relative gene expression data using real-time quantitative PCR and the 2(-delta delta C(T)) method. Methods 25: 402-408, 2001.

21. Lu J, Ji ML, Zhang XJ, Shi PL, Wu H, Wang $\mathrm{C}$ and $\mathrm{Im} \mathrm{HJ}$ : MicroRNA-218-5p as a potential target for the treatment of human osteoarthritis. Mol Ther 12: 2676-2688, 2017.

22. Balaskas P, Goljanek-Whysall K, Clegg P, Fang Y, Cremers A, Emans P, Welting T and Peffers M: MicroRNA profiling in cartilage ageing. Int J Genomics 2017: 2713725, 2017

23. Hong BK, You S, Yoo SA, Park D, Hwang D, Cho CS and Kim WU: MicroRNA-143 and -145 modulate the phenotype of synovial fibroblasts in rheumatoid arthritis. Exp Mol Med 49: e363, 2017.

24. Sümbül AT, Göğebakan B,Ergün S, Yengil E, Batmacı CY,TonyalıÖ and Yaldiz M: miR-204-5p expression in colorectal cancer: An autophagy-associated gene. Tumour Biol 35: 12713-12719, 2014.

25. Wu C, Tian B, Qu X, Liu F, Tang T, Qin A, Zhu Z and Dai K: MicroRNAs play a role in chondrogenesis and osteoarthritis (review). Int J Mol Med 34: 13-23, 2014

26. Wuelling $M$ and Vortkamp A: Chondrocyte proliferation and differentiation. Endocr Dev 21: 1-11, 2011.

27. Hosaka Y, Saito T, Sugita S, Hikata T, Kobayashi H, Fukai A, Taniguchi Y, Hirata M, Akiyama H, Chung UI and Kawaguchi H: Notch signaling in chondrocytes modulates endochondral ossification and osteoarthritis development. Proc Natl Acad Sci USA 110: 1875-1880, 2013.
28. Komori T, Yagi H, Nomura S, Yamaguchi A, Sasaki K, Deguchi K, Shimizu Y, Bronson RT, Gao YH, Inada M, et al: Targeted disruption of Cbfa1 results in a complete lack of bone formation owing to maturational arrest of osteoblasts. Cell 89: 755-764, 1997.

29. Otto F, Thornell AP, Crompton T, Denzel A, Gilmour KC, Rosewell IR, Stamp GW, Beddington RS, Mundlos S, Olsen BR, et al: Cbfal, a candidate gene for cleidocranial dysplasia syndrome, is essential for osteoblast differentiation and bone development. Cell 89: 765-771, 1997.

30. Lee YJ, Park SY, Lee SJ, Boo YC, Choi JY and Kim JE: Ucma, a direct transcriptional target of Runx 2 and Osterix, promotes osteoblast differentiation and nodule formation. Osteoarthritis Cartilage 23: 1421-1431, 2015.

31. Komori T: Regulation of skeletal development by the Runx family of transcription factors. J Cell Biochem 95: 445-453, 2005.

32. Hashimoto K, Oda Y, Nakamura F, Kakinoki R and Akagi M: Lectin-like, oxidized low-density lipoprotein receptor-1-deficient mice show resistance to age-related knee osteoarthritis. Eur J Histochem 61: 2762, 2017.

33. DeLise AM, Fischer L and Tuan RS: Cellular interactions and signaling in cartilage development. Osteoarthritis Cartilage 8: 309-334, 2000

34. Quarto R, Campanile G, Cancedda R and Dozin B: Modulation of commitment, proliferation, and differentiation of chondrogenic cells in defined culture medium. Endocrinology 138: 4966-4976, 1997.

35. Goldring MB, Tsuchimochi $\mathrm{K}$ and Ijiri K: The control of chondrogenesis. J Cell Biochem 97: 33-44, 2006.

36. Hesselstrand R, Kassner A, Heinegard D and Saxne T: COMP: A candidate molecule in the pathogenesis of systemic sclerosis with a potential as a disease marker. Ann Rheum Dis 67: 1242-1248, 2008.

This work is licensed under a Creative Commons Attribution-NonCommercial-NoDerivatives 4.0 International (CC BY-NC-ND 4.0) License. 